\title{
Degenerate polyexponential functions and type 2 degenerate poly-Bernoulli numbers and polynomials
}

\author{
Taekyun Kim ${ }^{1,2}$, Dae San Kim³ ${ }^{3}$ Jongkyum Kwon ${ }^{4 *}$ and Hyunseok Lee ${ }^{2}$
}

"Correspondence:

mathkjk26@gnu.ac.kr

${ }^{4}$ Department of Mathematics

Education and ERI, Gyeongsang

National University, Jinju, Korea

Full list of author information is

available at the end of the article

\section{Springer}

\begin{abstract}
The polyexponential functions were introduced by Hardy and rediscovered by Kim, as inverses to the polylogarithm functions. Recently, the type 2 poly-Bernoulli numbers and polynomials were defined by means of the polyexponential functions. In this paper, we introduce the degenerate polyexponential functions and the degenerate type 2 poly-Bernoulli numbers and polynomials, as degenerate versions of such functions and numbers and polynomials. We derive several explicit expressions and some identities for those numbers and polynomials.
\end{abstract}

MSC: 11B83;05A19

Keywords: Degenerate polyexponential functions; Type 2 degenerate poly-Bernoulli polynomials; Type 2 degenerate poly-Bernoulli numbers

\section{Introduction}

For $k \in \mathbb{Z}$, the polyexponential function is defined by

$$
\operatorname{Ei}_{k}(x)=\sum_{n=1}^{\infty} \frac{x^{n}}{(n-1) ! n^{k}} \quad(\text { see }[10])
$$

By (1), we see that $\operatorname{Ei}_{1}(x)=e^{x}-1$.

The polyexponential function was first introduced by Hardy and is given by

$$
e(x, a \mid s)=\sum_{n=0}^{\infty} \frac{x^{n}}{(n+a)^{s} n !} \quad(\operatorname{Re}(a)>0) .
$$

We note here that $e(x, 1 \mid k)=\frac{1}{x} \mathrm{Ei}_{k}(x)$.

In [10], the type 2 poly-Bernoulli polynomials are defined by

$$
\frac{1}{e^{t}-1} \mathrm{Ei}_{k}(\log (1+t)) e^{x t}=\sum_{n=0}^{\infty} B_{n}^{(k)}(x) \frac{t^{n}}{n !}
$$

When $x=0, B_{n}^{(k)}=B_{n}^{(k)}(0)$ are called type 2 poly-Bernoulli numbers.

(c) The Author(s) 2020. This article is licensed under a Creative Commons Attribution 4.0 International License, which permits use, sharing, adaptation, distribution and reproduction in any medium or format, as long as you give appropriate credit to the original author(s) and the source, provide a link to the Creative Commons licence, and indicate if changes were made. The images or other third party material in this article are included in the article's Creative Commons licence, unless indicated otherwise in a credit line to the material. If material is not included in the article's Creative Commons licence and your intended use is not permitted by statutory regulation or exceeds the permitted use, you will need to obtain permission directly from the copyright holder. To view a copy of this licence, visit http://creativecommons.org/licenses/by/4.0/. 
From (1) and (2), we note that $B_{n}^{(1)}(x)=B_{n}(x)(n \geq 0)$, where $B_{n}(x)$ are ordinary Bernoulli polynomials given by

$$
\frac{t}{e^{t}-1} e^{x t}=\sum_{n=0}^{\infty} B_{n}(x) \frac{t^{n}}{n !} \quad(\text { see }[1-18,20-24])
$$

In particular, $B_{n}=B_{n}(0)(n \geq 0)$ are called Bernoulli numbers.

For $\lambda \in \mathbb{R}$, the degenerate exponential function is defined as

$$
e_{\lambda}^{x}(t)=(1+\lambda t)^{\frac{x}{\lambda}}, \quad e_{\lambda}(t)=e_{\lambda}^{1}(t)=(1+\lambda t)^{\frac{1}{\lambda}} \quad(\text { see }[11-15,17,19]) .
$$

In $[2,3]$, Carlitz considered the degenerate Bernoulli polynomials which are given by

$$
\frac{t}{e_{\lambda}(t)-1} e_{\lambda}^{x}(t)=\frac{t}{(1+\lambda t)^{\frac{1}{\lambda}}-1}(1+\lambda t)^{\frac{x}{\lambda}}=\sum_{n=0}^{\infty} \beta_{n, \lambda}(x) \frac{t^{n}}{n !} .
$$

When $x=0, \beta_{n, \lambda}=\beta_{n, \lambda}(0)$ are called degenerate Bernoulli numbers.

Recently, the degenerate polylogarithm function was defined by Kim-Kim as

$$
l_{k, \lambda}(x)=\sum_{n=1}^{\infty} \frac{(-\lambda)^{n-1}(1)_{n, 1 / \lambda}}{(n-1) ! n^{k}} x^{n} \quad(k \in \mathbb{Z},|x|<1)(\text { see }[17])
$$

where $(x)_{0, \lambda}=1,(x)_{n, \lambda}=x(x-\lambda) \cdots(x-(n-1) \lambda)(n \geq 1)$.

Note that $\lim _{\lambda \rightarrow 0} l_{k, \lambda}(x)=\sum_{n=1}^{\infty} \frac{x^{n}}{n^{k}}=\mathrm{Li}_{k}(x)$ is the polylogarithm of index $k$.

For $k \in \mathbb{Z}$, the degenerate poly-Bernoulli numbers are defined by

$$
\left.\frac{1}{x} l_{k, \lambda}(x)\right|_{x=1-e_{\lambda}(-t)}=\sum_{n=0}^{\infty} \beta_{n, \lambda}^{(k)} \frac{t^{n}}{n !} \quad(\text { see [17]). }
$$

In [17], the degenerate Stirling numbers of the second kind are defined by

$$
(x)_{n, \lambda}=\sum_{l=0}^{n} S_{2, \lambda}(n, l)(x)_{l} \quad(n \geq 0) .
$$

As an inversion formula of (7), the degenerate Stirling numbers of the first kind are defined by

$$
(x)_{n}=\sum_{l=0}^{n} S_{1, \lambda}(n, l)(x)_{l, \lambda} \quad(n \geq 0)(\text { see }[23]) .
$$

From (7) and (8), we note that

$$
\frac{1}{k !}\left(e_{\lambda}(t)-1\right)^{k}=\sum_{n=k}^{\infty} S_{2, l}(n, k) \frac{t^{n}}{n !}
$$

and

$$
\frac{1}{k !}\left(\log _{\lambda}(1+t)\right)^{k}=\sum_{n=k}^{\infty} S_{1, \lambda}(n, k) \frac{t^{n}}{n !} \quad(k \geq 0)(\text { see }[17])
$$


where $\log _{\lambda}(t)=\frac{1}{\lambda}\left(t^{\lambda}-1\right)$ is the compositional inverse of $e_{\lambda}(t)$ satisfying $\log _{\lambda}\left(e_{\lambda}(t)\right)=$ $e_{\lambda}\left(\log _{\lambda}(t)\right)=t$.

Kaneko defined the poly-Bernoulli numbers by making use of the polylogarithm functions and Kim-Kim-Kim-Jang studied degenerate poly-Bernoulli numbers and polynomials by using polyexponential function [18]. The polyexponential functions were first introduced by Hardy and rediscovered recently by Kim-Kim [10], as inverses to the polylogarithm functions. In addition, the type 2 poly-Bernoulli numbers and polynomials were defined by means of the polyexponential functions. In this paper, we study the degenerate polyexponential functions and the degenerate type 2 poly-Bernoulli polynomials and numbers, as degenerate versions of such functions and numbers and polynomials. We derive several explicit expressions and some identities for those numbers and polynomials.

\section{Type 2 degenerate poly-Bernoulli numbers and polynomials}

The degenerate polyexponential function is defined in [15]. In the light of (1), we now consider the degenerate modified polyexponential function given by

$$
\operatorname{Ei}_{k, \lambda}(x)=\sum_{n=1}^{\infty} \frac{(1)_{n, \lambda} x^{n}}{(n-1) ! n^{k}} \quad(k \in \mathbb{Z},|x|<1) .
$$

Note that $\mathrm{Ei}_{1, \lambda}(x)=e_{\lambda}(x)-1$.

From (11), we note that

$$
\frac{d}{d x} \operatorname{Ei}_{k, \lambda}(x)=\frac{1}{x} \sum_{n=1}^{\infty} \frac{(1)_{n, \lambda} x^{n}}{(n-1) ! n^{k-1}}=\frac{1}{x} \operatorname{Ei}_{k-1, \lambda}(x) .
$$

For $k \geq 2$, by (12), we have

$$
\begin{aligned}
\operatorname{Ei}_{k, \lambda}(x) & =\int_{0}^{x} \underbrace{\frac{1}{t} \int_{0}^{t} \frac{1}{t} \int_{0}^{t} \cdots \frac{1}{t} \int_{0}^{t} \frac{1}{t}}_{(k-2) \text {-times }} \operatorname{Ei}_{1, \lambda}(t) d t \cdots d t \\
& =\int_{0}^{x} \underbrace{\frac{1}{t} \int_{0}^{t} \frac{1}{t} \int_{0}^{t} \cdots \frac{1}{t} \int_{0}^{t} \frac{1}{t}\left(e_{\lambda}(t)-1\right) d t \cdots d t}_{(k-2) \text {-times }} .
\end{aligned}
$$

In view of (2) and using the degenerate modified polyexponential function, we define the type 2 degenerate poly-Bernoulli polynomials by

$$
\frac{\operatorname{Ei}_{k, \lambda}\left(\log _{\lambda}(1+t)\right)}{e_{\lambda}(t)-1} e_{\lambda}^{x}(t)=\sum_{n=0}^{\infty} B_{n, \lambda}^{(k)}(x) \frac{t^{n}}{n !} \quad(k \in \mathbb{Z}) .
$$

When $x=0, B_{n, \lambda}^{(k)}=B_{n, \lambda}^{(k)}(0)$ are called type 2 degenerate poly-Bernoulli numbers.

It is well known that the degenerate Bernoulli polynomials of the second kind are defined by

$$
\frac{t}{\log _{\lambda}(1+t)}(1+t)^{x}=\sum_{n=0}^{\infty} b_{n, \lambda}(x) \frac{t^{n}}{n !} \quad(\text { see [17]). }
$$


When $x=0, b_{n, \lambda}=b_{n, \lambda}(0)(n \geq 0)$, are called degenerate Bernoulli numbers of the second kind.

Note that $\lim _{\lambda \rightarrow 0} b_{n, \lambda}=b_{n}(n \geq 0)$. Here $b_{n}$ are the Bernoulli numbers of the second kind, according to Roman [23], given by

$$
\frac{t}{\log (1+t)}=\sum_{n=0}^{\infty} b_{n} \frac{t^{n}}{n !} \quad(\text { see }[6,22,23]) .
$$

From (12), we note that

$$
\begin{aligned}
\frac{d}{d x} \operatorname{Ei}_{k, \lambda}\left(\log _{\lambda}(1+x)\right) & =\frac{d}{d x} \sum_{n=1}^{\infty} \frac{(1)_{n, \lambda}\left(\log _{\lambda}(1+x)\right)^{n}}{(n-1) ! n^{k}} \\
& =\frac{(1+x)^{\lambda-1}}{\log _{\lambda}(1+x)} \sum_{n=1}^{\infty} \frac{(1)_{n, \lambda}\left(\log _{\lambda}(1+x)\right)^{n}}{(n-1) ! n^{k-1}} \\
& =\frac{(1+x)^{\lambda-1}}{\log _{\lambda}(1+x)} \operatorname{Ei}_{k-1, \lambda}\left(\log _{\lambda}(1+x)\right) .
\end{aligned}
$$

By (17), for $k \geq 2$, we have

$$
\operatorname{Ei}_{k, \lambda}\left(\log _{\lambda}(1+x)\right)=\int_{0}^{x} \underbrace{\frac{(1+t)^{\lambda-1}}{\log _{\lambda}(1+t)} \int_{0}^{t} \cdots \frac{(1+t)^{\lambda-1}}{\log _{\lambda}(1+t)} \int_{0}^{t}}_{(k-2) \text {-times }} \frac{(1+t)^{\lambda-1}}{\log _{\lambda}(1+t)} t d t \cdots d t
$$

Thus, from (14) and (18), we have

$$
\begin{aligned}
& \sum_{n=0}^{\infty} B_{n, \lambda}^{(k)} \frac{x^{n}}{n !}=\frac{1}{e_{\lambda}(x)-1} \operatorname{Ei}_{k, \lambda}\left(\log _{\lambda}(1+x)\right) \\
& =\frac{1}{e_{\lambda}(x)-1} \int_{0}^{x} \underbrace{\frac{(1+t)^{\lambda-1}}{\log _{\lambda}(1+t)} \int_{0}^{t} \cdots \frac{(1+t)^{\lambda-1}}{\log _{\lambda}(1+t)} \int_{0}^{t}}_{(k-2) \text {-times }} \frac{(1+t)^{\lambda-1}}{\log _{\lambda}(1+t)} t d t \cdots d t \\
& =\frac{x}{e_{\lambda}(x)-1} \sum_{m=0}^{\infty} \sum_{m_{1}+\cdots+m_{k-1}=m}\left(\begin{array}{c}
m \\
m_{1}, \ldots, m_{k-1}
\end{array}\right) \\
& \times \frac{b_{m_{1}, \lambda}(\lambda-1)}{m_{1}+1} \frac{b_{m_{2}, \lambda}(\lambda-1)}{m_{1}+m_{2}+1} \cdots \frac{b_{m_{k-1}, \lambda}(\lambda-1)}{m_{1}+\cdots+m_{k-1}+1} \frac{x^{m}}{m !} \\
& =\sum_{n=0}^{\infty} \sum_{m=0}^{n}\left(\begin{array}{c}
n \\
m
\end{array}\right) \sum_{m_{1}+\cdots+m_{k-1}=m}\left(\begin{array}{c}
m \\
m_{1}, \ldots, m_{k-1}
\end{array}\right) \\
& \times \frac{b_{m_{1}, \lambda}(\lambda-1)}{m_{1}+1} \frac{b_{m_{2}, \lambda}(\lambda-1)}{m_{1}+m_{2}+1} \cdots \frac{b_{m_{k-1}, \lambda}(\lambda-1)}{m_{1}+\cdots+m_{k-1}+1} \beta_{n-m, \lambda} \frac{x^{n}}{n !} .
\end{aligned}
$$

Therefore, by (19), we obtain the following theorem. 
Theorem 1 For $n \geq 0$, we have

$$
\begin{aligned}
B_{n, \lambda}^{(k)}= & \sum_{m=0}^{n}\left(\begin{array}{c}
n \\
m
\end{array}\right) \sum_{m_{1}+\cdots+m_{k-1}=m}\left(\begin{array}{c}
m \\
m_{1}, \ldots, m_{k-1}
\end{array}\right) \\
& \times \frac{b_{m_{1}, \lambda}(\lambda-1)}{m_{1}+1} \frac{b_{m_{2}, \lambda}(\lambda-1)}{m_{1}+m_{2}+1} \cdots \frac{b_{m_{k-1}, \lambda}(\lambda-1)}{m_{1}+\cdots+m_{k-1}+1} \beta_{n-m, \lambda} .
\end{aligned}
$$

From (14), we note that

$$
\begin{aligned}
\sum_{n=0}^{\infty} B_{n, \lambda}^{(k)}(x) \frac{t^{n}}{n !} & =\frac{\operatorname{Ei}_{k, \lambda}\left(\log _{\lambda}(1+t)\right)}{e_{\lambda}(t)-1} e_{\lambda}^{x}(t) \\
& =\sum_{l=0}^{\infty} B_{l, \lambda}^{(k)} \frac{t^{l}}{l !} \sum_{m=0}^{\infty}(x)_{m, \lambda} \frac{t^{m}}{m !} \\
& =\sum_{n=0}^{\infty}\left(\sum_{l=0}^{n}\left(\begin{array}{l}
n \\
l
\end{array}\right)(x)_{n-l, \lambda} B_{l, \lambda}^{(k)}\right) \frac{t^{n}}{n !} .
\end{aligned}
$$

Therefore, by comparing the coefficients on both sides of (21), we obtain the following theorem.

Theorem 2 For $n \geq 0$, we have

$$
B_{n, \lambda}^{(k)}(x)=\sum_{l=0}^{n}\left(\begin{array}{l}
n \\
l
\end{array}\right)(x)_{n-l, \lambda} B_{l, \lambda}^{(k)} .
$$

Now, we observe that

$$
\begin{aligned}
\frac{1}{e_{\lambda}(t)-1} \operatorname{Ei}_{k, \lambda}\left(\log _{\lambda}(1+t)\right) & =\frac{1}{e_{\lambda}(t)-1} \sum_{m=1}^{\infty} \frac{(1)_{m, \lambda}\left(\log _{\lambda}(1+t)\right)^{m}}{(m-1) ! m^{k}} \\
& =\frac{1}{e_{\lambda}(t)-1} \sum_{m=1}^{\infty} \frac{(1)_{m, \lambda}}{m^{k-1}} \frac{1}{m !}\left(\log _{\lambda}(1+t)\right)^{m} \\
& =\frac{1}{e_{\lambda}(t)-1} \sum_{m=1}^{\infty} \frac{(1)_{m, \lambda}}{m^{k-1}} \sum_{n=m}^{\infty} S_{1, \lambda}(n, m) \frac{t^{n}}{n !} \\
& =\frac{1}{e_{\lambda}(t)-1} \sum_{n=1}^{\infty}\left(\sum_{m=1}^{n} \frac{(1)_{m, \lambda}}{m^{k-1}} S_{1, \lambda}(n, m)\right) \frac{t^{n}}{n !} \\
& =\frac{t}{e_{\lambda}(t)-1} \sum_{n=0}^{\infty} \frac{1}{n+1}\left(\sum_{m=1}^{n+1} S_{1, \lambda}(n+1, m) \frac{(1)_{m, \lambda}}{m^{k-1}}\right) \frac{t^{n}}{n !}
\end{aligned}
$$

Thus, by (4) and (22), we get

$$
\begin{aligned}
\frac{1}{e_{\lambda}(t)-1} \operatorname{Ei}_{k, \lambda}\left(\log _{\lambda}(1+t)\right) & =\sum_{j=0}^{\infty} \beta_{j, \lambda} \frac{t^{j}}{j !} \sum_{l=0}^{\infty} \frac{1}{l+1}\left(\sum_{m=1}^{l+1} \frac{S_{1, \lambda}(l+1, m)(1)_{m, \lambda}}{m^{k-1}}\right) \frac{t^{l}}{l !} \\
& =\sum_{n=0}^{\infty}\left(\sum_{l=0}^{n} \frac{\left(\begin{array}{l}
n \\
l
\end{array}\right)}{l+1} \sum_{m=1}^{l+1} \frac{S_{1, \lambda}(l+1, m)}{m^{k-1}}(1)_{m, \lambda} \beta_{n-l, \lambda}\right) \frac{t^{n}}{n !}
\end{aligned}
$$


On the other hand,

$$
\frac{1}{e_{\lambda}(t)-1} \operatorname{Ei}_{k, \lambda}\left(\log _{\lambda}(1+t)\right)=\sum_{n=0}^{\infty} B_{n, \lambda}^{(k)} \frac{t^{n}}{n !}
$$

Therefore, by (23) and (24), we obtain the following theorem.

Theorem 3 For $n \geq 0$, we have

$$
B_{n, \lambda}^{(k)}=\sum_{l=0}^{n} \frac{\left(\begin{array}{c}
n \\
l
\end{array}\right)}{l+1} \sum_{m=1}^{l+1} \frac{S_{1, \lambda}(l+1, m)}{m^{k-1}}(1)_{m, \lambda} \beta_{n-l, \lambda}
$$

By letting $\lambda \rightarrow 0$, we get

$$
B_{n}^{(k)}=\sum_{l=0}^{n} \frac{\left(\begin{array}{l}
n \\
l
\end{array}\right)}{l+1} \sum_{m=1}^{l+1} \frac{S_{1}(l+1, m)}{m^{k-1}} B_{n-l} \quad(n \geq 0) .
$$

From (14), we note that

$$
\begin{aligned}
\operatorname{Ei}_{k, \lambda}\left(\log _{\lambda}(1+t)\right) & =\left(e_{\lambda}(t)-1\right) \sum_{l=0}^{\infty} B_{l, \lambda}^{(k)} \frac{t^{l}}{l !} \\
& =\left(\sum_{m=0}^{\infty} \frac{(1)_{m, \lambda}}{m !} t^{m}-1\right) \sum_{l=0}^{\infty} B_{l, \lambda}^{(k)} \frac{t^{l}}{l !} \\
& =\sum_{n=0}^{\infty}\left(\sum_{m=0}^{n}\left(\begin{array}{c}
n \\
m
\end{array}\right)(1)_{n-m, \lambda} B_{m, \lambda}^{(k)}-B_{n, \lambda}^{(k)}\right) \frac{t^{n}}{n !} \\
& =\sum_{n=1}^{\infty}\left(B_{n, \lambda}^{(k)}(1)-B_{n, \lambda}^{(k)}\right) \frac{t^{n}}{n !} .
\end{aligned}
$$

On the other hand,

$$
\begin{aligned}
\operatorname{Ei}_{k, \lambda}\left(\log _{\lambda}(1+t)\right) & =\sum_{m=1}^{\infty} \frac{(1)_{m, \lambda}\left(\log _{\lambda}(1+t)\right)^{m}}{(m-1) ! m^{k}} \\
& =\sum_{m=1}^{\infty} \frac{(1)_{m, \lambda}}{m^{k-1}} \frac{1}{m !}\left(\log _{\lambda}(1+t)\right)^{m} \\
& =\sum_{m=1}^{\infty} \frac{(1)_{m, \lambda}}{m^{k-1}} \sum_{n=m}^{\infty} S_{1, \lambda}(n, m) \frac{t^{n}}{n !} \\
& =\sum_{n=1}^{\infty}\left(\sum_{m=1}^{n} \frac{(1)_{m, \lambda} S_{1, \lambda}(n, m)}{m^{k-1}}\right) \frac{t^{n}}{n !}
\end{aligned}
$$

Therefore, by (25) and (26), we obtain the following theorem.

Theorem 4 For $n \in \mathbb{N}$ and $k \in \mathbb{Z}$, we have

$$
B_{n, \lambda}^{(k)}(1)-B_{n, \lambda}^{(k)}=\sum_{m=1}^{n} \frac{(1)_{m, \lambda} S_{1, \lambda}(n, m)}{m^{k-1}} .
$$


From (11), we note that

$$
\operatorname{Ei}_{1, \lambda}(x)=\sum_{n=1}^{\infty} \frac{(1)_{n, \lambda}}{n !} x^{n}=e_{\lambda}(x)-1
$$

By (27), we get

$$
\begin{aligned}
\operatorname{Ei}_{1, \lambda}\left(\log _{\lambda}(1+t)\right) & =\sum_{m=1}^{\infty} \frac{(1)_{m, \lambda}}{m !}\left(\log _{\lambda}(1+t)\right)^{m} \\
& =\sum_{m=1}^{\infty}(1)_{m, \lambda} \sum_{n=m}^{\infty} S_{1, \lambda}(n, m) \frac{t^{n}}{n !} \\
& =\sum_{n=1}^{\infty}\left(\sum_{m=1}^{n}(1)_{m, \lambda} S_{1, \lambda}(n, m)\right) \frac{t^{n}}{n !} .
\end{aligned}
$$

On the other hand,

$$
\operatorname{Ei}_{1, \lambda}\left(\log _{\lambda}(1+t)\right)=t
$$

Therefore, by (28) and (29), we obtain the following theorem.

Theorem 5 For $n \in \mathbb{N}$, we have

$$
\sum_{m=1}^{n}(1)_{m, \lambda} S_{1, \lambda}(n, m)=\delta_{n, 1}
$$

where $\delta_{n, k}$ is the Kronecker delta.

Note that

$$
\lim _{\lambda \rightarrow 0}\left(B_{n, \lambda}^{(1)}(1)-B_{n, \lambda}^{(1)}\right)=B_{n}(1)-B_{n}= \begin{cases}1, & \text { if } n=1, \\ 0, & \text { if } n>1 .\end{cases}
$$

Thus, by Theorems 4 and 5, we get

$$
\beta_{n, \lambda}(1)-\beta_{n, \lambda}=\sum_{m=1}^{n}(1)_{m, \lambda} S_{1, \lambda}(n, m)= \begin{cases}1, & \text { if } n=1, \\ 0, & \text { if } n>1 .\end{cases}
$$

From (14), we note that

$$
\frac{\operatorname{Ei}_{k, \lambda}\left(\log _{\lambda}(1+t)\right)}{e_{\lambda}(t)-1}=\sum_{n=0}^{\infty} B_{n, \lambda}^{(k)} \frac{t^{n}}{n !}
$$


By replacing $t$ by $e_{\lambda}(t)-1$, we get

$$
\begin{aligned}
\sum_{m=0}^{\infty} & B_{m, \lambda}^{(k)} \frac{1}{m !}\left(e_{\lambda}(t)-1\right)^{m} \\
& =\frac{\operatorname{Ei}_{k, \lambda}(t)}{e_{\lambda}\left(e_{\lambda}(t)-1\right)-1}=\frac{e_{\lambda}(t)-1}{e_{\lambda}\left(e_{\lambda}(t)-1\right)-1} \frac{t}{e_{\lambda}(t)-1} \frac{1}{t} \operatorname{Ei}_{k, \lambda}(t) \\
& =\sum_{i_{1}=0}^{\infty} \beta_{i_{1}, \lambda} \frac{1}{i_{1} !}\left(e_{\lambda}(t)-1\right)^{i_{1}} \sum_{j=0}^{\infty} \beta_{j, \lambda} \frac{t^{j}}{j !} \sum_{m=0}^{\infty} \frac{(1)_{m+1, \lambda}}{(m+1)^{k}} \frac{t^{m}}{m !} \\
& =\sum_{i_{1}=0}^{\infty} \beta_{i_{1}, \lambda} \sum_{i_{2}=i_{1}}^{\infty} S_{2, \lambda}\left(i_{2}, i_{1}\right) \frac{t^{i_{2}}}{i_{2} !} \sum_{j=0}^{\infty} \beta_{j, \lambda} \frac{t^{j}}{j !} \sum_{m=0}^{\infty} \frac{(1)_{m+1, \lambda}}{(m+1)^{k}} \frac{t^{m}}{m !} \\
& =\sum_{i_{2}=0}^{\infty} \sum_{i_{1}=0}^{i_{2}} \beta_{i_{1}, \lambda} S_{2, \lambda}\left(i_{2}, i_{1}\right) \frac{t^{i_{2}}}{i_{2} !} \sum_{j=0}^{\infty} \beta_{j, \lambda} \frac{t^{j}}{j !} \sum_{m=0}^{\infty} \frac{(1)_{m+1, \lambda}}{(m+1)^{k}} \frac{t^{m}}{m !} \\
& =\sum_{i_{3}=0}^{\infty}\left(\sum_{i_{2}=0}^{i_{3}} \sum_{i_{1}=0}^{i_{2}}\left(\begin{array}{l}
i_{3} \\
i_{2}
\end{array}\right) \beta_{i_{1}, \lambda} S_{2, \lambda}\left(i_{2}, i_{1}\right) \beta_{i_{3}-i_{2}, \lambda}\right) \frac{t^{i_{3}}}{i_{3} !} \sum_{m=0}^{\infty} \frac{(1)_{m+1, \lambda}}{(m+1)^{k}} \frac{t^{m}}{m !} \\
= & \sum_{n=0}^{\infty}\left(\sum_{i_{3}=0}^{n} \sum_{i_{2}=0}^{i_{3}} \sum_{i_{1}=0}^{i_{2}}\left(\begin{array}{l}
n \\
i_{3}
\end{array}\right)\left(\begin{array}{l}
i_{3} \\
i_{2}
\end{array}\right) \beta_{i_{1}, \lambda} S_{2, \lambda}\left(i_{2}, i_{1}\right) \beta_{i_{3}-i_{2}, \lambda} \frac{(1)_{n-i_{3}+1, \lambda}}{\left(n-i_{3}+1\right)^{k}}\right) \frac{t^{n}}{n !}
\end{aligned}
$$

On the other hand,

$$
\begin{aligned}
\sum_{m=0}^{\infty} B_{m, \lambda}^{(k)} \frac{1}{m !}\left(e_{\lambda}(t)-1\right)^{m} & =\sum_{m=0}^{\infty} B_{m, \lambda}^{(k)} \sum_{n=m}^{\infty} S_{2, \lambda}(n, m) \frac{t^{n}}{n !} \\
& =\sum_{n=0}^{\infty}\left(\sum_{m=0}^{n} B_{m, \lambda}^{(k)} S_{2, \lambda}(n, m)\right) \frac{t^{n}}{n !}
\end{aligned}
$$

Therefore, by (32) and (33), we obtain the following theorem.

\section{Theorem 6}

$$
\sum_{m=0}^{n} B_{m, \lambda}^{(k)} S_{2, \lambda}(n, m)=\sum_{i_{3}=0}^{n} \sum_{i_{2}=0}^{i_{3}} \sum_{i_{1}=0}^{i_{2}}\left(\begin{array}{l}
n \\
i_{3}
\end{array}\right)\left(\begin{array}{l}
i_{3} \\
i_{2}
\end{array}\right) \beta_{i_{1}, \lambda} S_{2, \lambda}\left(i_{2}, i_{1}\right) \beta_{i_{3}-i_{2}, \lambda} \frac{(1)_{n-i_{3}+1, \lambda}}{\left(n-i_{3}+1\right)^{k}}
$$

\section{Further remark}

The higher-order degenerate Bernoulli polynomials are defined by Carlitz and given by

$$
\left(\frac{t}{e_{\lambda}(t)-1}\right)^{r} e_{\lambda}^{x}(t)=\sum_{n=0}^{\infty} \beta_{n, \lambda}^{(r)}(x) \frac{t^{n}}{n !} \quad(\text { see }[2,3])
$$

where $r$ is a positive integer.

When $x=0, \beta_{n, \lambda}^{(r)}=\beta_{n, \lambda}^{(r)}(0)$ are called higher-order degenerate Bernoulli numbers. 
We observe that

$$
\begin{aligned}
\frac{1}{t^{r}}\left(\log _{\lambda}(1+t)\right)^{r} & =\frac{r !}{t^{r}} \frac{1}{r !}\left(\log _{\lambda}(1+t)\right)^{r} \\
& =\frac{r !}{t^{r}} \sum_{n=r}^{\infty} S_{1, \lambda}(n, r) \frac{t^{n}}{n !} \\
& =\frac{r !}{t^{r}} \sum_{n=0}^{\infty} S_{1, \lambda}(n+r, r) \frac{n !}{(n+r) !} \frac{t^{n+r}}{n !} \\
& =\sum_{n=0}^{\infty} \frac{S_{1, \lambda}(n+r, r)}{\left(\begin{array}{c}
n+r \\
r
\end{array}\right)} \frac{t^{n}}{n !} .
\end{aligned}
$$

Replacing $t$ by $\log _{\lambda}(1+t)$ in (34), we get

$$
\begin{aligned}
\left(\frac{\log _{\lambda}(1+t)}{t}\right)^{r} & =\sum_{m=0}^{\infty} \beta_{m, \lambda}^{(r)} \frac{1}{m !}\left(\log _{\lambda}(1+t)\right)^{m} \\
& =\sum_{m=0}^{\infty} \beta_{m, \lambda}^{(r)} \sum_{n=m}^{\infty} S_{1, \lambda}(n, m) \frac{t^{n}}{n !} \\
& =\sum_{n=0}^{\infty}\left(\sum_{m=0}^{n} \beta_{m, \lambda}^{(r)} S_{1, \lambda}(n, m)\right) \frac{t^{n}}{n !} .
\end{aligned}
$$

Therefore, by (35) and (36), we obtain the following theorem.

Theorem 7 For $n \geq 0$, we have

$$
S_{1, \lambda}(n+r, r)=\left(\begin{array}{c}
n+r \\
r
\end{array}\right) \sum_{m=0}^{n} \beta_{m, \lambda}^{(r)} S_{1, \lambda}(n, m) .
$$

Now, we consider the inversion formula of Theorem 7. Replacing $t$ by $e_{\lambda}(t)-1$ in (35), we get

$$
\begin{aligned}
\left(\frac{t}{e_{\lambda}(t)-1}\right)^{r} & =\sum_{m=0}^{\infty} \frac{S_{1}(m+r, r)}{\left(\begin{array}{c}
m+r \\
r
\end{array}\right)} \frac{1}{m !}\left(e_{\lambda}(t)-1\right)^{m} \\
& =\sum_{m=0}^{\infty} \frac{S_{1}(m+r, r)}{\left(\begin{array}{c}
m+r \\
r
\end{array}\right)} \sum_{n=m}^{\infty} S_{2, \lambda}(n, m) \frac{t^{n}}{n !} \\
& =\sum_{n=0}^{\infty}\left(\sum_{m=0}^{n} \frac{S_{1, \lambda}(m+r, r)}{\left(\begin{array}{c}
m+r \\
r
\end{array}\right)} S_{2, \lambda}(n, m)\right) \frac{t^{n}}{n !} .
\end{aligned}
$$

On the other hand,

$$
\left(\frac{t}{e_{\lambda}(t)-1}\right)^{r}=\sum_{n=0}^{\infty} \beta_{n, \lambda}^{(r)} \frac{t^{n}}{n !} \quad(\text { see }[2,3]) .
$$

Therefore, by (37) and (38), we obtain the following theorem. 
Theorem 8 For $n \geq 0$, we have

$$
\beta_{n, \lambda}^{(r)}=\sum_{m=0}^{n} \frac{S_{1, \lambda}(m+r, m)}{\left(\begin{array}{c}
m+r \\
r
\end{array}\right)} S_{2, \lambda}(n, m) .
$$

Replacing $t$ by $\log _{\lambda}(1+t)$ in (9) and making use of (10), we get

$$
\begin{aligned}
\frac{1}{k !} t^{k} & =\sum_{m=k}^{\infty} S_{2, \lambda}(m, k) \frac{1}{m !}\left(\log _{\lambda}(1+t)\right)^{m} \\
& =\sum_{m=k}^{\infty} S_{2, \lambda}(m, k) \sum_{n=m}^{\infty} S_{1, \lambda}(n, m) \frac{t^{n}}{n !} \\
& =\sum_{n=k}^{\infty}\left(\sum_{m=k}^{n} S_{2, \lambda}(m, k) S_{1, \lambda}(n, m)\right) \frac{t^{n}}{n !} .
\end{aligned}
$$

Therefore, by comparing the coefficient on both sides of (39), we get

$$
\sum_{m=k}^{n} S_{1, \lambda}(n, m) S_{2, \lambda}(m, k)=\delta_{n, k} \quad(0 \leq k \leq n)
$$

where $\delta_{n, k}$ is Kronecker's delta.

The degenerate Bernoulli numbers of the second kind of order $r$ are given by

$$
\left(\frac{t}{\log _{\lambda}(1+t)}\right)^{r}=\sum_{n=0}^{\infty} b_{n, \lambda}^{(r)} \frac{t^{n}}{n !}
$$

Note that $\lim _{\lambda \rightarrow 0} b_{n, \lambda}^{(r)}=b_{n}^{(r)}$ are the Bernoulli numbers of the second kind of order $r$.

Replacing $t$ by $e_{\lambda}(t)-1$, we get

$$
\begin{aligned}
\frac{1}{t^{r}}\left(e_{\lambda}(t)-1\right)^{r} & =\sum_{m=0}^{\infty} b_{m, \lambda}^{(r)} \frac{1}{m !}\left(e_{\lambda}(t)-1\right)^{m} \\
& =\sum_{m=0}^{\infty} b_{m, \lambda}^{(r)} \sum_{n=m}^{\infty} S_{2, \lambda}(n, m) \frac{t^{n}}{n !} \\
& =\sum_{n=0}^{\infty}\left(\sum_{m=0}^{n} b_{m, \lambda}^{(r)} S_{2, \lambda}(n, m)\right) \frac{t^{n}}{n !} .
\end{aligned}
$$

On the other hand,

$$
\begin{aligned}
\frac{1}{t^{r}}\left(e_{\lambda}(t)-1\right)^{r} & =\frac{r !}{t^{r}} \frac{1}{r !}\left(e_{\lambda}(t)-1\right)^{r}=\frac{r !}{t^{r}} \sum_{n=r}^{\infty} S_{2, \lambda}(n, r) \frac{t^{n}}{n !} \\
& =\sum_{n=0}^{\infty} \frac{S_{2, \lambda}(n+r, r)}{\left(\begin{array}{c}
n+r \\
r
\end{array}\right)} \frac{t^{n}}{n !} .
\end{aligned}
$$


Thus, by (42) and (43), we get

$$
S_{2, \lambda}(n+r, r)=\left(\begin{array}{c}
n+r \\
r
\end{array}\right) \sum_{m=0}^{n} b_{m, \lambda}^{(r)} S_{2, \lambda}(n, m) .
$$

By the same method as in the above, the inversion formula of (44) is given by

$$
b_{n}^{(r)}=\sum_{m=0}^{n} \frac{S_{2, \lambda}(m+r, r)}{\left(\begin{array}{c}
m+r \\
r
\end{array}\right)} S_{1, \lambda}(n, m) \quad(n \geq 0) .
$$

\section{Conclusion}

Recently, Kim-Kim introduced degenerate polyexponential functions and degenerate Bell polynomials [15] and they studied degenerate poly-Bernoulli numbers and polynomials from degenerate polylogarithm function. In [10], Kim-Kim also studied polyexponential functions as an inverse to the polylogarithm functions, constructed type 2 poly-Bernoulli polynomials by using this and derived various properties of type 2 poly-Bernoulli numbers. In addition, they investigated unipoly functions attached to each suitable arithmetic function as a universal concept which includes the polylogarithm and polyexponential functions as special cases. As the degenerate version of the type 2 poly-Bernoulli polynomials, we study the degenerate polyexponential functions and the degenerate type 2 poly-Bernoulli numbers and polynomials. Finally, we derive several explicit expressions and some identities for those numbers and polynomials. Proof techniques and results developed in this research paper are expected to be of great help to researchers in this field in the future.

\section{Acknowledgements}

We thank the reviewers for their valuable comments and suggestions. In addition, the authors would like to thank Jangjeon Institute for Mathematical Science for the support of this research.

Funding

This work was supported by the National Research Foundation of Korea (NRF)grant funded by the Korea government (MEST) (No. 2017R1E1A1A03070882).

Availability of data and materials

Not applicable.

Competing interests

The authors declare to have no conflict of interest.

Authors' contributions

TK and DSK conceived of the framework and structured the whole paper; DSK and TK wrote the paper; JK and HL checked the results of the paper and typed the paper; DSK and TK completed the revision of the article. All authors have read and agreed with the published version of the manuscript.

\section{Author details}

${ }^{1}$ School of Science, Xi'an Technological University, Xi'an, China. ${ }^{2}$ Department of Mathematics, Kwangwoon University, Seoul, Republic of Korea. ${ }^{3}$ Department of Mathematics, Sogang University, Seoul, Republic of Korea. ${ }^{4}$ Department of Mathematics Education and ERI, Gyeongsang National University, Jinju, Korea.

\section{Publisher's Note}

Springer Nature remains neutral with regard to jurisdictional claims in published maps and institutional affiliations. 


\section{References}

1. Araci, S., Acikgoz, M.: A note on the Frobenius-Euler numbers and polynomials associated with Bernstein polynomials. Adv. Stud. Contemp. Math. (Kyungshang) 22(3), 399-406 (2012)

2. Carlitz, L.: A degenerate Staudt-Clausen theorem. Arch. Math. (Basel) 7, 28-33 (1956)

3. Carlitz, L.: Degenerate Stirling, Bernoulli and Eulerian numbers. Util. Math. 15, 51-88 (1979)

4. Chung, S.-K., Jang, G.-W., Kim, D.S., Kwon, J.: Some identities of the type 2 degenerate Bernoulli and Euler numbers. Adv. Stud. Contemp. Math. (Kyungshang) 29(4), 613-632 (2019)

5. Jang, L.-C., Ryoo, C.S., Lee, J.G., Kwon, H.I.: On the k-th degeneration of the Genocchi polynomials. J. Comput. Anal. Appl. 22(7), 1343-1349 (2017)

6. Jeong, J., Rim, S.-H., Kim, B.M.: On finite-times degenerate Cauchy numbers and polynomials. Adv. Differ. Equ. 2015, Article ID 321 (2015)

7. Jeong, W.K.: Some identities for degenerate cosine(sine)-Euler polynomials. Adv. Stud. Contemp. Math. (Kyungshang) 30(1), 155-164 (2020)

8. Khan, W.A., Ahmad, M.: Partially degenerate poly-Bernoulli polynomials associated with Hermite polynomials. Adv. Stud. Contemp. Math. (Kyungshang) 28(3), 487-496 (2018)

9. Kim, T., Kim, D.S., Dolgy, D.V., Kwon, J.: Some identities on generalized degenerate Genocchi and Euler numbers. Informatica 31(4), 42-51 (2020)

10. Kim, D.S., Kim, T.: A note on polyexponential and unipoly functions. Russ. J. Math. Phys. 26(1), 40-49 (2019)

11. Kim, D.S., Kim, T., Lee, H., Kwon, J.: A note on $\lambda$-Bernoulli numbers of the second kind. Adv. Stud. Contemp. Math. (Kyungshang) 30(2) (2020, in press)

12. Kim, T.: A note on degenerate Stirling polynomials of the second kind. Proc. Jangjeon Math. Soc. 20(3), 319-331 (2017)

13. Kim, T., Kim, D.S.: Degenerate Laplace transform and degenerate gamma function. Russ. J. Math. Phys. 24(2), 241-248 (2017)

14. Kim, T., Kim, D.S.: A note on central Bell numbers and polynomials. Russ. J. Math. Phys. 27(1), 76-81 (2020)

15. Kim, T., Kim, D.S.: Degenerate polyexponential functions and degenerate Bell polynomials. J. Math. Anal. Appl. 487(2), $124017(2020)$

16. Kim, T., Kim, D.S.: Some identities of extended degenerate r-central Bell polynomials arising from umbral calculus Rev. R. Acad. Cienc. Exactas Fís. Nat., Ser. A Mat. 114(1), Art. 1 (2020)

17. Kim, T., Kim, D.S.: A note on new type degenerate Bernoulli numbers. Russ. Math. Phys. (accepted)

18. Kim, T., Kim, D.S., Kim, H.Y., Jang, L.-C.: Degenerate poly-Bernoulli numbers and polynomials. Informatica 31(3), 2-8 (2020)

19. Kim, T., Kim, D.S., Lee, H., Kwon, J.: Degenerate binomial coefficients and degenerate hypergeometric functions. Adv. Differ. Equ. 2020, Article ID 115 (2020)

20. Kruchinin, D.V., Kruchinin, V.V.: Explicit formula for reciprocal generating function and its application. Adv. Stud. Contemp. Math. (Kyungshang) 29(3), 365-372 (2019)

21. Ma, M., Lim, D.: Some identities on the fully degenerate Bell polynomials of the second kind. Adv. Stud. Contemp. Math. (Kyungshang) 30(1), 145-154 (2020)

22. Pyo, S.-S.: Degenerate Cauchy numbers and polynomials of the fourth kind. Adv. Stud. Contemp. Math. (Kyungshang) 28(1), 127-138 (2018)

23. Roman, S.: The Umbral Calculus. Pure and Applied Mathematics, vol. 111. Academic Press, New York (1984)

24. Simsek, Y.: Identities on the Changhee numbers and Apostol-type Daehee polynomials. Adv. Stud. Contemp. Math (Kyungshang) 27(2), 199-212 (2017)

\section{Submit your manuscript to a SpringerOpen ${ }^{\odot}$ journal and benefit from:}

- Convenient online submission

- Rigorous peer review

- Open access: articles freely available online

- High visibility within the field

- Retaining the copyright to your article

Submit your next manuscript at $>$ springeropen.com 